\title{
Prevalência e perfil de sensibilidade de bactérias isoladas da urina de gestantes atendidas no serviço de obstetrícia de um hospital terciário
}

\author{
Prevalence and susceptibility profile of bacteria isolated from urine of \\ pregnant women seen at the department of obstetrics of a tertiary hospital \\ Fernanda Aguirre Carvalho', Mônica de Abreu Rodrigues ${ }^{1}$, Angelita Bottega', Rosmari Hörner ${ }^{2} \bowtie$ \\ 1 Programa de Pós-Graduação em Ciências Farmacêuticas, Universidade Federal de Santa Maria. Santa Maria, RS. \\ ${ }^{2}$ Departamento de Análises Clínicas e Toxicológicas do Centro de Ciências da Saúde, Universidade Federal de Santa Maria. Santa Maria, RS.
}

\section{RESUMO}

Objetivos: Avaliar a prevalência e o perfil de sensibilidade aos antimicrobianos de bactérias isoladas de uroculturas de gestantes atendidas no ambulatório do Serviço de Obstetrícia do Hospital Universitário de Santa Maria, Brasil.

Métodos: Foi realizado um estudo retrospectivo dos laudos emitidos pelo Laboratório de Análises Clínicas do Hospital Universitário de Santa Maria. Foram incluídas no estudo todas as uroculturas positivas de gestantes atendidas no ambulatório do Serviço de Obstetrícia deste hospital, no período de janeiro a dezembro de 2014. Os testes de identificação dos micro-organismos isolados e os perfis de sensibilidade frente aos antimicrobianos foram efetuados por meio de um sistema automatizado.

Resultados: No período do estudo foram identificadas neste laboratório 423 uroculturas positivas provenientes de gestantes. A bactéria Gram negativa Escherichia coli foi a mais prevalente (46,50\% das culturas positivas). A segunda bactéria mais frequente foi a Gram positiva Staphylococcus saprophyticus (6,2\%). O fungo Candida spp. foi isolado de $94(21,8 \%)$ amostras de urina. Nitrofurantoína e amoxicilina/ácido clavulânico foram os antimicrobianos com menor taxa de resistência por parte de E. coli ( $91,33 \%$ e 90,77\% de sensibilidade, respectivamente). Já frente às bactérias Gram positivas prevalentes, ampicilina foi a que mostrou maior sensibilidade.

Conclusões: O perfil de suscetibilidade apresentado neste estudo indica que a escolha para o tratamento da ITU na gestação pode recair em nitrofurantoína e/ou amoxicilina/ácido clavulânico para as bactérias Gram negativas. Tendo em vista a prevalência encontrada, esses antimicrobianos podem ser iniciados empiricamente antes do resultado da urocultura, nos casos de ITU sintomática. Este estudo ratifica, entretanto, a importância da realização da urocultura entre os exames pré-natais e sua repetição no terceiro trimestre da gravidez, tendo em vista a variedade de micro-organismos identificados e os diferentes perfis de sensibilidade aos antimicrobianos testados.

DESCRITORES: infecções urinárias; urocultura; gestação; testes de sensibilidade microbiana.

\section{ABSTRACT}

Aims: To evaluate the prevalence of bacteria in urine cultures of pregnant women seen at the outpatient clinic of the Department of Obstetrics at the University Hospital of Santa Maria, Brazil, and to determine the antibiotic sensitivity profile of these bacteria.

Methods: The reports issued by the Laboratory of Clinical Analysis of the University Hospital of Santa Maria were retrospectively analyzed. All positive urine cultures of pregnant women seen at the Department of Obstetrics from January to December 2014 were included in the study. The tests for the identification of bacterial isolates and their sensitivity profiles were assessed by an automated system.

Results: A total of 423 positive urine cultures were detected in the pregnant women. Gram-negative Escherichia coli was the most prevalent microorganism (46.50\%). Gram-positive Staphylococcus saprophyticus was the second most prevalent bacterium (6.2\%). Candida spp. was isolated from $94(21.8 \%)$ urine samples. Nitrofurantoin and amoxicillin/clavulanic acid showed the lowest antimicrobial resistance against E. coli $(91.33 \%$ and $90.77 \%$, respectively). Ampicillin had the highest sensitivity among prevalent Gram-positive bacteria.

Conclusions: The sensitivity profile found in this study allows us to suggest nitrofurantoin and/or amoxicillin/clavulanic acid for the treatment of urinary tract infection caused by Gram-negative bacteria during pregnancy. Given the prevalence rates detected in this study, these antimicrobials can be initiated empirically before the urine culture results are known, in the cases of symptomatic urinary tract infection. This study underscores the importance of urine culture in the prenatal period and in the third trimester because of the different microorganisms identified and the different sensitivity to the antimicrobials tested.

KEY WORDS: urinary tract infections; urine culture; pregnancy; microbial sensitivity tests. 
Abreviaturas: CLSI, Clinical and Laboratory Standards Institute; HUSM, Hospital Universitário de Santa Maria; ITU, infecção do trato urinário; UFC, unidades formadoras de colônia.

\section{INTRODUÇÃO}

As infecções do trato urinário (ITU) representam o segundo grupo de doenças infecciosas mais comum na prática clínica, apenas menos frequente que o grupo de infecções do trato respiratório. As ITU perfazem 30 a $50 \%$ das infeções relacionadas à assistência à saúde, acometendo principalmente crianças e mulheres. São divididas em infecções do trato urinário alto, que são as ureterites e pielonefrites, e infecções do trato urinário baixo, nas mulheres cistite ou uretrite e nos homens mais comumente prostatite ou epididimite. A bacteriúria assintomática é abordada junto com as ITU, mas constitui-se em um quadro particular, com abordagem e prognóstico diferentes [1].

$\mathrm{Na}$ gravidez, as ITU são as infecções bacterianas mais frequentes, estando associadas a índices de morbidade e mortalidade materna e perinatal significativas. Essas infecções são mais comuns no período gestacional devido às mudanças anatômicas, hormonais e fisiológicas impostas que ocorrem nesse período. $\mathrm{Na}$ bacteriúria assintomática, a gravidez é a única indicação absoluta de tratamento, pois as gestantes com essa condição têm um risco 20 a 30 vezes maior de desenvolver pielonefrite. A pielonefrite aguda é uma das principais indicações de hospitalização durante a gestação [1-6].

As complicações da ITU na gestação incluem trabalho de parto prematuro, rotura prematura de membranas ovulares, febre no pós-parto, baixo peso ao nascer, sepse materna e infecção neonatal [2]. Estudo realizado por Mata et al. [7], em Alagoas, encontrou 57,7\% de partos prematuros em gestantes com ITU [8]. Para a prevenção dessas complicações, é importante o diagnóstico precoce, bem como a identificação do microorganismo envolvido e seu perfil de suscetibilidade aos antimicrobianos, que conduzirão ao tratamento adequado $[2,8]$. Dentre as bactérias envolvidas em ITU, destaca-se Escherichia coli, responsável por 80$85 \%$ dos casos. Outros agentes etiológicos frequentes das ITU são Staphylococcus saprophyticus, Proteus mirabilis, Streptococcus agalactiae e Klebsiella spp. As ITU podem ser causadas também, esporadicamente, por fungos do gênero Candida spp [3,9].
O tratamento de ITU em gestantes deve ser feito imediatamente após o diagnóstico, antes mesmo da confirmação do micro-organismo e do resultado do antibiograma, devido aos riscos que essa infecção pode trazer à gestante e ao feto. Por isso ressalta-se a importância de avaliação periódica do padrão de sensibilidade dos micro-organismos causadores de ITU [8].

Em razão da toxicidade de alguns fármacos para o feto, existe a restrição de antibióticos para gestantes, reduzindo assim o arsenal terapêutico [8]. Todos os agentes pertencentes à classe das quinolonas devem ser evitados, exceto em situações onde não exista outro antimicrobiano potencialmente útil. A associação sulfametoxazol/trimetoprima não é recomendada no primeiro trimestre, bem como no último mês da gestação (pelo risco da sulfa causar hiperbilirrubinemia no recém-nascido que tiver deficiência da enzima glicose-6-fosfato desidrogenase) [10].

Os antimicrobianos que podem ser utilizados com segurança na gravidez são cefalexina (cefalosporina de primeira geração), ampicilina, amoxicilina e nitrofurantoína, sendo que o último deve ser usado com cautela no último trimestre da gravidez $[10,11]$. Outras opções incluem algumas cefalosporinas de segunda ou terceira geração [12]. No caso de pielonefrite, o tratamento recomendado é por via parenteral em nível hospitalar $[8,11]$.

Devido à importância de identificar precocemente a ITU e iniciar o tratamento antes do resultado da urocultura e do antibiograma, o objetivo deste estudo foi avaliar a prevalência de uropatógenos, bem como avaliar o perfil de sensibilidade aos antimicrobianos em uroculturas de gestantes atendidas no ambulatório do Serviço de Obstetrícia do Hospital Universitário de Santa Maria (HUSM), no período de janeiro a dezembro de 2014.

\section{MÉTODOS}

Foi realizado um estudo retrospectivo, que avaliou os laudos de uroculturas positivas de gestantes, realizadas no período compreendido entre janeiro e dezembro de 2014. Foram incluídos no estudo todos os laudos microbiológicos positivos das uroculturas de gestantes atendidas no ambulatório do Serviço de Obstetrícia do HUSM, mesmo que alguns desses exames fossem realizados pela mesma paciente, em ocasiões diferentes.

Todos os exames foram processados pelo Laboratório de Análises Clínicas do HUSM. Foram consideradas culturas positivas as placas que apresentaram 
contagem de unidades formadoras de colônia (UFC) por mililitro $(\mathrm{mL})$ de urina não centrifugada maior ou igual a $10^{5} \mathrm{UFC} / \mathrm{mL}$, de acordo com as recomendações do Clinical and Laboratory Standards Institute (CLSI) [13].

Em relação ao padrão de sensibilidade aos antimicrobianos, foram analisadas para este estudo somente as cinco bactérias predominantes no período estudado. Os testes de identificação das bactérias isoladas e os perfis de sensibilidade frente aos antimicrobianos foram efetuados por meio do sistema automatizado Vitek ${ }^{\circledR} 2$ (BioMérieux, Marcy I'Etoile, França), seguindo-se as recomendações do CLSI [13].

Utilizaram-se cartões para Gram negativo em que foram testados os antimicrobianos ampicilina, amoxicilina/ácido clavulânico, piperacilina/tazobactam, cefalotina, cefuroxima, cefuroxima axetil, ceftriaxona, cefepima, ertapenem, meropenem, amicacina, gentamicina, ácido nalidíxico, ciprofloxacina, norfloxacina, nitrofurantoína e sulfametoxazol/trimetoprima. Utilizaram-se também, cartões para Gram postivo em que foi testada a sensibilidade frente a benzilpenicilina, ampicilina, oxacilina, gentamicina alto nível, estreptomicina alto nível, gentamicina, ciprofloxacina, moxifloxacina, norfloxacina, eritromicina, clindamicina, linezolida, teicoplanina, vancomicina, tigeciclina, ácido fusídico, rifampicina e sulfametoxazol/trimetoprima.

Este estudo é parte de um projeto maior intitulado "Identificação e avaliação da sensibilidade aos antimicrobianos e mecanismos de resistência de bactérias isoladas no hospital universitário de Santa Maria", tendo sido aprovado pelo Comitê de Ética em Pesquisa da Universidade Federal de Santa Maria, sob o número 38850614.4.0000.5346.

\section{RESULTADOS}

No período em estudo, 2.328 uroculturas realizadas no laboratório de Análises Clínicas do HUSM resultaram positivas. Destas, 432 (18,5\%) foram de gestantes atendidas no ambulatório do Serviço de Obstetrícia do HUSM, cujas idades variaram entre 14 e 55 anos (média de 25,8 88,05 anos). Não foram coletados dados clínicos das pacientes e não foram discriminados os exames correspondentes a casos de bacteriúria assintomática ou de ITU sintomática. Entre as 432 uroculturas positivas, 240 (55,5\%) corresponderam a bactérias Gram negativas, 98 $(22,7 \%)$ a bactérias Gram positivas e $94(21,8 \%)$ ao fungo Candida spp.
Entre as bactérias isoladas, E.coli foi a predominante $(197 / 432 ; 45,6 \%)$, seguida por $S$. saprophyticus (27/432; 6,2\%), Enterococcus faecalis (19/432; 4,4\%), K. pneumoniae $(19 / 432 ; 4,4 \%)$ e $S$. agalactiae $(18 / 432$; $4,2 \%)$. As demais bactérias juntas corresponderam a $17,8 \%$ do total de isolamentos. A positividade para Candida spp não foi concomitante com a positividade para bactérias (Tabela 1).

Tabela 1. Prevalência de micro-organismos em uroculturas de gestantes atendidas no ambulatório do Serviço de Obstetrícia do Hospital Universitário de Santa Maria, Brasil, no período de janeiro a dezembro de 2014.

\begin{tabular}{lcc}
\hline \multirow{2}{*}{ Bactéria } & \multicolumn{2}{c}{ Prevalência } \\
\cline { 2 - 3 } & $\mathbf{n}$ & $(\%)$ \\
\hline Escherichia coli & 197 & $(45,60)$ \\
Staphylococcus saprophyticus & 27 & $(6,25)$ \\
\hline Enterococcus faecalis & 19 & $(4,40)$ \\
\hline Klebsiella pneumoniae & 19 & $(4,40)$ \\
\hline Streptococcus agalactiae & 18 & $(4,17)$ \\
\hline Staphylococcus aureus & 14 & $(3,24)$ \\
Proteus mirabilis & 10 & $(2,31)$ \\
\hline Staphylococcus hominis & 7 & $(1,62)$ \\
Enterococcus aerogenes & 6 & $(1,39)$ \\
Staphylococcus epidermidis & 6 & $(1,39)$ \\
Staphylococcus haemolyticus & 3 & $(0,69)$ \\
Staphylococcus warneri & 3 & $(0,69)$ \\
Citrobacter koseri & 2 & $(0,46)$ \\
\hline Staphylococcus ludgunensis & 1 & $(0,23)$ \\
Candida spp & 94 & $(21,76)$ \\
Total & 432 & $(100,00)$ \\
\hline
\end{tabular}

As cinco bactérias predominantes no período estudado foram analisadas em relação à sensibilidade aos antimicrobianos. Duas delas eram Gram negativas (E. coli e $K$. pneumoniae), correspondendo a 216 uroculturas, e três eram Gram positivas (S. saprophyticus, E. faecalis e S. agalactiae), correspondendo a 64 uroculturas.

E. coli apresentou alta resistência para ampicilina (31\%) e sulfametoxazol/trimetoprima $(22 \%)$ e boa sensibilidade a amoxicilina/ácido clavulânico e nitrofurantoina (90,8 e 91,3\% respectivamente), medicamentos passíveis de uso na gravidez (Tabela 2).

Entre os principais patógenos Gram positivos, $E$. faecalis e $S$. agalactiae apresentaram boa sensibilidade frente à ampicilina (79\% e $100 \%$, respectivamente). $S$. saprophyticus foi o micro-organismo que apresentou as maiores taxas de resistência frente aos antimicrobianos (Tabela 3). 
Tabela 2. Perfil de sensibilidade das bactérias Gram negativas mais frequentemente isoladas $(n=216)$ em uroculturas de gestantes atendidas no ambulatório do Serviço de Obstetrícia do Hospital Universitário de Santa Maria, Brasil, no período de janeiro a dezembro de 2014.

\begin{tabular}{|c|c|c|c|c|c|c|}
\hline \multirow{2}{*}{$\begin{array}{c}\text { Agente } \\
\text { antimicrobiano }\end{array}$} & \multicolumn{3}{|c|}{$\begin{array}{l}\text { Escherichia coli } \\
\quad(n=197)\end{array}$} & \multicolumn{3}{|c|}{$\begin{array}{l}\text { Klebsiella pneumoniae } \\
\qquad(\mathrm{n}=19)\end{array}$} \\
\hline & S (\%) & I (\%) & $\mathrm{R}(\%)$ & S (\%) & I (\%) & $\mathbf{R}(\%)$ \\
\hline Ácido nalidíxico & 89,8 & 0 & 10,2 & 84,6 & 0 & 15,4 \\
\hline AMC & 90,8 & 5,1 & 4,1 & 100 & 0 & 0 \\
\hline Amicacina & 98,5 & 0,5 & 1,0 & 100 & 0 & 0 \\
\hline Ampicilina & 66,5 & 2,5 & 31,0 & 0 & 0 & 100 \\
\hline Cefalotina & 66,3 & 22,0 & 11,7 & 84,6 & 15,4 & 0 \\
\hline Cefepima & 97,0 & 0 & 3,0 & 100 & 0 & 0 \\
\hline Cefetriaxana & 96,4 & 0 & 3,6 & 100 & 0 & 0 \\
\hline Cefuroxima & 90,4 & 4,0 & 5,6 & 84,6 & 15,4 & 0 \\
\hline Cefuroxima Axetil & 87,8 & 6,6 & 5,6 & 84,6 & 15,4 & 0 \\
\hline Ciprofloxacina & 95,4 & 0 & 4,6 & 100 & 0 & 0 \\
\hline Ertapenem & 100 & 0 & 0 & 100 & 0 & 0 \\
\hline Gentamicina & 93,9 & 0 & 6,1 & 100 & 0 & 0 \\
\hline Meropenem & 98,5 & 0 & 1,5 & 100 & 0 & 0 \\
\hline Nitrofurantoína & 91,3 & 5,1 & 3,6 & 53,8 & 23,1 & 23,1 \\
\hline Norfloxacina & 97,0 & 0,5 & 2,5 & 100 & 0 & 0 \\
\hline SXT & 78,0 & 0 & 22,0 & 100 & 0 & 0 \\
\hline TZP & 89,5 & 7,3 & 3,2 & 91,7 & 0 & 8,3 \\
\hline
\end{tabular}

AMC, amoxicilina/ácido clavulânico; SXT, sulfametoxazol/trimetoprima; TZP, piperacilina/tazobactam; S, suscetível; I, intermediário; R, resistente.

\section{DISCUSSÃO}

Levando em consideração os riscos que a ITU pode trazer tanto para a gestante quanto para o concepto, este estudo analisou a prevalência e o padrão de sensibilidade dos principais uropatógenos isolados de gestantes que buscaram atendimento no Serviço de Obstetrícia do HUSM.

E. coli foi o micro-organismo mais prevalente no presente estudo $(45,6 \%)$, dado este que está de acordo com o de outras pesquisas realizadas no Brasil, diferindo apenas nas porcentagens. Outros estudos encontraram frequências muito maiores (80-85\%) [3,14-17]. Pode-se justificar essa alta prevalência pelo fato da bactéria fazer parte da flora intestinal normal, o que a torna um dos principais agentes envolvidos em casos de bacteriúria assintomática em gestantes [18].

Em relação à sensibilidade frente aos antimicrobianos, E. coli apresentou perfil semelhante a outros estudos, com taxas de resistência à ampicilina e sulfametoxazol/trimetoprima de $31 \%$ e $21,9 \%$, (as quais foram as maiores taxas de resistência), situação esta que pode ser resultante da ampla utilização desses medicamentos nos últimos anos $[3,14]$.

Tabela 3. Perfil de sensibilidade das bactérias Gram positivas mais frequentemente isoladas $(n=64)$ em uroculturas de gestantes atendidas no ambulatório do Serviço de Obstetrícia do Hospital Universitário de Santa Maria, Brasil, no período de janeiro a dezembro de 2014.

\begin{tabular}{|c|c|c|c|c|c|c|c|c|c|}
\hline \multirow{2}{*}{$\begin{array}{c}\text { Agente } \\
\text { antimicrobiano }\end{array}$} & \multicolumn{3}{|c|}{$\begin{array}{l}\text { Staphylococcus saprophyticus } \\
\qquad(\mathrm{n}=27)\end{array}$} & \multicolumn{3}{|c|}{$\begin{array}{l}\text { Enterococcus faecalis } \\
\qquad(\mathrm{n}=19)\end{array}$} & \multicolumn{3}{|c|}{$\begin{array}{l}\text { Streptococcus agalactiae } \\
(\mathrm{n}=18)\end{array}$} \\
\hline & S (\%) & I (\%) & R (\%) & S (\%) & I (\%) & R (\%) & S (\%) & I (\%) & R (\%) \\
\hline Ácido fusídico & 14,8 & 85,2 & 0 & 100 & 0 & 0 & - & - & - \\
\hline Ampicilina & - & - & - & 79,0 & 0 & 21,1 & 100 & 0 & 0 \\
\hline Benzilpenicilina & 3,7 & 0 & 96,3 & - & - & - & 100 & 0 & 0 \\
\hline Ciprofloxacina & 0 & 0 & 100 & - & - & - & 94,4 & 0 & 5,6 \\
\hline Clindamicina & 74,1 & 7,4 & 18,5 & 31,6 & 57,9 & 10,5 & 66,7 & 0 & 33,3 \\
\hline Eritromicina & 74,1 & 3,7 & 22,2 & - & - & - & 66,7 & 5,6 & 27,8 \\
\hline Estreptomicina & - & - & - & 94,7 & 0 & 5,3 & - & - & - \\
\hline Gentamicina & 0 & 0 & 100 & 89,5 & 0 & 10,5 & - & - & - \\
\hline Linezolida & 0 & 0 & 100 & 0 & 0 & 100 & 100 & 0 & 0 \\
\hline Moxifloxacina & 0 & 0 & 100 & 94,7 & 5,3 & 0 & 94,4 & 0 & 5,6 \\
\hline Norfloxacina & 0 & 0 & 100 & 0 & 0 & 100 & 0 & 50,0 & 50,0 \\
\hline Oxacilina & 59,3 & 0 & 40,7 & 79,0 & 0 & 21,1 & - & - & - \\
\hline Rifampicina & 92,3 & 7,7 & 0 & - & - & - & - & - & - \\
\hline SXT & 69,2 & 0 & 30,8 & - & - & - & - & - & - \\
\hline Teicoplanina & 0 & 0 & 100 & 94,7 & 5,3 & 0 & 100 & 0 & 0 \\
\hline Tigeciclina & 96,3 & 0 & 3,7 & 100 & 0 & 0 & 100 & 0 & 0 \\
\hline Vancomicina & 96,3 & 0 & 3,7 & 100 & 0 & 0 & 100 & 0 & 0 \\
\hline
\end{tabular}

SXT, sulfametoxazol/trimetoprima; S, suscetível; I, intermediário; R, resistente. 
Frente à cefalotina, (cefalosporina de primeira geração, que deve ser evitada na gestação), a sensibilidade foi baixa $(66,3 \%)$, dado este que difere do estudo feito por Pereira et al. [14], na cidade de Palmas, que encontrou $93 \%$ de sensibilidade frente a esse antibiótico. A sensibilidade à cefalexina, outra cefalosporina de primeira geração e que é permitida na gestação, não foi analisada por não estar incluída na rotina do laboratório, sendo esta uma limitação do presente estudo.

Cefalosporinas de primeira geração têm sido amplamente utilizadas e recomendadas no tratamento empírico de infecções urinárias, e este fato pode ser uma justificativa para surgimento de resistência bacteriana [3]. Estudo realizado por Borges et al. [16] encontrou grande resistência das bactérias a essa classe farmacêutica.

Qualquer tratamento farmacológico de gestantes precisa levar em conta o risco de teratogênese, com o que, o arsenal terapêutico fica restrito. Como boa opção de tratamento, entre os antimicrobianos possíveis de ser utilizados na gestação, obteve-se a amoxilina/ácido clavulânico, corroborando com estudos que encontraram altas taxas de sensibilidade a esse antibiótico [14].

Candida spp representou $21,8 \%$ das uroculturas positivas em gestantes no período do estudo, sendo que as pacientes que apresentaram Candida spp não apresentaram outro microrganismo na urocultura. Constitui-se num fungo oportunista, que devido às alterações estruturais e anatômicas características da gestação, como o aumento de glicogênio e alterações no $\mathrm{pH}$, encontra condições favoráveis para causar a infecção [19].

Mesmo não sendo o micro-organismo prevalente em ITU de gestantes, deve-se dar grande importância à colonização por $S$. agalactiae (18/432; 4,2\%), devido à provável contaminação dos neonatos. Na gestante, S. agalactiae pode provocar, além de ITU, amnionite, endometrite e bacteremia. No recém-nascido pode causar infecção precoce, já na primeira semana de vida, ou mais tardia, entre a segunda e a quarta semanas de vida, sendo responsável por quadros graves, como septicemia, meningite e pneumonia. É importante salientar que o tratamento da gestante com ITU por $S$. agalactiae não dispensa a pesquisa dessa bactéria por swab vaginal e retal entre 35 e 37 semanas de gestação e a administração de penicilina intraparto [20].

Em conclusão, o perfil de suscetibilidade apresentado neste estudo indica que a escolha para o tratamento da ITU na gestação pode recair em nitrofurantoína e/ou amoxicilina/ácido clavulânico para as bactérias Gram negativas. Tendo em vista a prevalência encontrada, esses antimicrobianos podem ser iniciados empiricamente antes do resultado da urocultura, nos casos de ITU sintomática. Este estudo ratifica, entretanto, a importância da realização da urocultura entre os exames pré-natais e sua repetição no terceiro trimestre da gravidez, tendo em vista a variedade de micro-organismos identificados e os diferentes perfis de sensibilidade aos antimicrobianos testados.

\section{NOTA}

Declaração de conflitos de interesse

Os autores declaram não haver conflitos de interesse relevantes ao conteúdo deste estudo.

\section{REFERÊNCIAS}

1. Ministério da Saúde (BR). Agência Nacional de Vigilância Sanitária - ANVISA. Manual de Microbiologia Clínica para o Controle de Infecção Relacionada à Assistência a Saúde. Módulo 3: Principais Síndromes Infecciosas. Brasília; 2013 [cited 2015 Dec 27 ]. Available from: http://www20.anvisa.gov.br/segurancadopaciente/index.php/publicacoes/item/principais-sindromes-infecciosas

2. Figueiredo A, Gomes G, Campos A. Urinary tract infections in pregnancy - diagnosis treatment and prevention. Acta Obstet Ginecol Port. 2012;6(3):124-33.

3. Schenkel DF, Dallé J, Antonello VS. Microbial etiology and susceptibility of community urinary tract infections during pregnancy in the South of Brazil. Rev Bras Ginecol Obstet. 2014;36(3):102-6. http://dx.doi.org/10.1590/S0100-72032014000300002

4. Hackenhaar AA, Albernaz EP. Prevalência e fatores associados à internação hospitalar para tratamento da infecção do trato urinário durante a gestação. Rev Bras Ginecol Obstet. 2013;35(5):199-204. http://dx.doi.org/10.1590/S0100-72032013000500002

5. Castro DM, Gonçalves J, Braga JS. Pielonefrite aguda na gravidez complicada por síndrome de dificuldade respiratória aguda - a propósito de dois casos clínicos. Acta Obstet Ginecol Port .2015;9(2):174-8.

6. Dawkins JC, Fletcher HM, Rattray CA, Reid M, Gordon-Strachan G. Acute pyelonephritis in pregnancy: A retrospective descriptive hospital based-study. ISRN Obstet Gynecol. 2012;2012:519321. http://dx.doi.org/10.5402/2012/519321

7. Mata KS, Santos AAP, Oliveira e Silva JM, Holanda JBL, Silva FCL. Complications caused by urinary tract infection during pregnancy. Espaç Saúde. 2014;15(4):57-63. 
8. Duarte G, Marcolin AC, Quintana SM, Cavalli RC. Urinary tract infection in pregnancy. Rev Bras Ginecol Obstet. 2008;30(2):93-100.

9. Alós JI. Epidemiology and etiology of urinary tract infections in the community. Antimicrobial susceptibility of the main pathogens and clinical significance of resistance. Enferm Infecc Microbiol Clin. 2005;23(4):3-8.

10. Lopes HV, Tavares W; Associação Médica Brasileira; Conselho Federal de Medicina Projeto Diretrizes. Cistites em situações especiais: tratamento. CFM; 2004.

11. Heilberg IP, Schor N. Abordagem diagnóstica e terapêutica na infecção do trato urinário - Itu. Rev Assoc Med Bras. 2003;49(1):109-16. http://dx.doi.org/10.1590/S0104-42302003000100043

12. Fernandes FA, Oliveira CNT, Souza CL, Oliveira MV. Relevância do diagnóstico e tratamento da infecção do trato urinário em gestantes: uma revisão da literatura. C\&D. 2015;8(1):54-70.

13. Clinical and Laboratory Standards Institute (CLSI). Performance Standards for Antimicrobial Susceptibility Testing; Twenty-Fourth Informational Supplement, Document M100-S24.Wayne: CLSI; 2014;34(1):1-230.

14. Pereira AC, Bordignon JC. Urinary intection in pregnants: susceptibility profile of etiological agentes in pregnants followed by SUS in the city of Palmas-PR. Rev Bras Anal Clin. 2011;43(2):96-9.

15. Guerra GVQL, Souza ASR, Costa BF, Nascimento FRQ, Amaral MA, Serafim ACP. Urine test to diagnose urinary tract infection in highh-risk pregant women. Rev Bras Ginecol Obstet. 2012;34(11):488-93. http://dx.doi.org/10.1590/S0100-72032012001100002

16. Borges AA, Magalhães LG, Jabus APL, Cardoso AM. Infecção urinária em gestantes atendidas em laboratório clínico de Goiânia-GO entre 2012 e 2013. Estudos. 2014;41(3):637-48.

17. Ramos GC, Laurentino AP, Fochesatto S, Francisquetti FA, Rodrigues AD. Prevalência da trato urinário em gestantes em uma cidade no sul do Brasil. Saúde (Sta Maria). 2016;42(1):173-8.

18. Andabati G, Byamugisha J. Microbial aetiology and sesnsitivity of asymptomatic bacteriuria among ante-natal mothers in Mulago hospital, Uganda. Afr Health Sci. 2010;10(4):349-52.

19. Figueiró-Filho EA, Bispo AMB, Vasconcelos MM, Maia MZ, Celestino FG. Urinary tract infection in pregnancy: atual concepts. FEMINA. 2009;37(3):165-71.

20. Areal A, Nunes S, Moreira M, Faustino MA, Cardoso L, Sá C. Infecção perinatal por Streptococcus agalactiae pode ser evitada: Prevalência da colonização em parturientes no Hospital São Marcos, factores de risco e a sua relação com a infecção perinatal. Acta Pediatr Port. 2010;41(1):16-21. 